ausspricht. $\mathrm{Ob}$ dieses an langsameren Schwingungen verhältnissmässig reichere wiedergeweckte Phosphorescenzlicht von kürzerer oder längerer Dauer ist, würde davon abhängen, ob die betreffende Substanz langsamere Schwingungen nur vorübergehend oder aber dauernd auszuführen im Stande ist.

Weitere Ergebnisse der vorstehend beschriebenen Beobachtungsmethoden mögen späterer Mittheilung vorbehalten bleiben.

Erlangen, im October 1883.

VIII. Veber das specifische Gewicht des flüssigen Sauerstoffs; von Sigmund von Wroblewski. . (Im Auszuge mitgetheilt der Paris. Acad. d. Wiss., den 16. Juli 1883.)

(Hierzu Taf. VIII Fig. 1.)

$\S 1$. Die im Jahre 1877 ausgeführten Versuche von Raoul Pictet gaben die erste Veranlassung zur Berechnung des specifischen Gewichtes des flüssigen Sauerstoffes. In einem an $\mathrm{Pictet}$ gerichteten Brief hatte $\mathrm{Dumas}{ }^{3}$ ) die Vermuthung ausgesprochen, dass die Dichtigkeit des festen Sauerstoffes gleich eins und diejenige des flüssigen nicht viel verschieden davon sein könne. Betrachtet man nämlich Sauerstoff und Schwefel a.ls isomorphe Körper, so müssen die Quotienten aus den Atomgewichten in die specifischen Gewichte (oder - wie sie Dumas nennt - "volumes atomiques") bei beiden Körpern gleich sein. Da bei Schwefel dieser Quotient gleich $32 / 2=16$ ist, so müsse die Dichtigkeit des Sauerstoffes gleich $16=1$ sein.

Darauf glaubte Pictet die Zahlen 0,98S3 und 0,9787, die nur wenig von eins verschieden sind, aus seinen Versuchen ableiten zu können. Doch machte Offret²) bald darauf aufmerksam, dass diese Rechnung nicht richtig sein

1) R. Pictet, Ann. de chim. et de phys. (5) 13. p. 197-98. 1878.

2) Offret, Ann. de chim. et de phys. (5) 19. p. 271-283. 1880. 
könne, sondern dass sich die Zahl 0,84 als der wahrscheinlichste Werth ergebe.

Leider aber ist auch dieser Zahl kein grosses Vertrauen zu schenken. In dem Apparate von Pictet konnte der flüssige Sauerstoff nur in der durch die verdunstende Kohlensäure abgekühlten kupfernen Röhre sich sammeln. In der in Verbindung mit ihr stehenden eisernen Retorte, die zur Entwickelung des Gases diente und immer auf einer Temperatur von etwa $485^{\circ} \mathrm{C}$. gehalten wurde, konnte dagegen keine Verflüssigung eintreten. Die von Offret ausgeführte Berechnung der Dichtigkeit der Flüssigkeit hängt deshalb wesentlich von der Berechnung der zu subtrahirenden, im gasförmigen Zustande gebliebenen Sauerstoffmenge $a b$, und diese letztere kann nicht exact berechnet werden. Dazu fehlen: erstens die Kenntniss der Gesetze, welche das Mariotte-Gay-Lussac'sche Gesetz für den Sauerstoff in so grossen Temperaturgrenzen wie +485 und $-130^{\circ} \mathrm{C}$. ergänzen, zweitens die Kenntniss der Temperaturvertheilung und folglich der Dichtigkeitsvertheilung des Gases an der Uebergangsstelle der Röhre, wo der Temperaturwechsel mehr als $600^{\circ} \mathrm{C}$. beträgt. Ausserdem kann man nicht mit Sicherheit wissen, bis zu welcher Stelle die Röhre mit der Flüssigkeit gefüllt gewesen ist.

Ein anderer Weg zur Bestimmung der Dichtigkeit des flüssigen Sauerstoffes ist neulich von Cailletet und Hautefeuille ${ }^{1}$ ) eingeschlagen worden. Cailletet und Hautefeuille mischten ein Volumen Sauerstoff mit sieben Volumina Kohlensäure, erwärmten das Gemisch auf mehr als $31^{\circ} \mathrm{C}$., um die Ausscheidung der flüssigen Kohlensäure zu verhindern, und brachten es unter sehr hohen Druck. Als nachher die Temperatur in überaus gleichmässiger Weise erniedrigt wurde, verflüssigte sich das Gemisch wie ein einfaches Gas. Da die bei dieser Verflüssigung eintretende Contraction - wie dies aus Versuchen mit anderen Gasen hervorging - nur eine sehr geringe gewesen sein konnte,

1) Cailletet und Hautefenille, Compt. rend. 92. p. 1086-1090. 1881. 
so war man im Stande aus der Dichtigkeit der erhaltenen Flüssigkeit und der bekannten Dichtigkeit der Kohlensäure diejenige des Sauerstoffes annähernd zu berechnen. Die Resultate der Versuche sind in folgender Tabelle zusammen. gestellt:

Druck in Atmosphären $\quad . \quad \overbrace{200275}^{\text {bei } 0^{0} \mathrm{C} .} \frac{\overbrace{200}}{\text { bei }-23^{\circ} \mathrm{C} .}$

Dichtigkeit des flüssigen Sauer$\begin{array}{lllllll}\text { stoffes im Gemisch . . . . } & 0,58 & 0,65 & 0,70 & 0,84 & 0,88 & 0,89\end{array}$

Aus einem unter dem Drucke von 300 Atmosphären ausgeführten Versuche mit einem Gemisch ron Sauerstoff und Stickstoffoxydul ergab sich als Dichtigkeit bei $0^{\circ} \mathrm{C}$. 0,65 und bei $-23^{\circ} \mathrm{C} .0,94$.

§ 2. Zur Bestimmung des specifischen Gewichtes des flüssigen Sauerstoffes diente mein bereits in der Abhandlung „über die Verflüssigung des Sauerstoffes u. s. w." beschriebener Apparat. $\left.{ }^{1}\right)$ Der Deutlichkeit wegen wird hier in der Fig. 1 die aus der a. a. O. publicirten Abbildung des Apparates entnommene Capillare $q$ im vergrösserten Maassstabe wiedergegeben.

Auf diese Capillare ist die Millimetertheilung eingeätzt, und sie wurde darauf calibrirt. Ihr Volumen wurde genau ermittelt, ebenso das des Rohres $i$. Während die Füllung mit dem Gase ausgeführt wurde, befand sich das Rohr in einem Wasserbade. Der am unteren Ende des Rohres angesetzte Glashahn ${ }^{2}$ ) gestattete es, die Füllung genau unter dem atmosphärischen Drucke auszuführen. Aus dem Barometerstande und der Temperatur des $\mathrm{W}$ asserbades konnte deshalb die in das Rohr eingelassene Gasmenge exact berechnet werden. Die Länge des nach abwärts gebogenen Theiles der Capillare wurde so gewählt, dass, wenn der Sauerstoff verflüssigt wurde, und man mit dem Quecksilber bis zur Biegungsstelle $A$ der Capillare kam, der Meniskus des verflüssigten Gases ein paar Centimeter unter dem Kautschukpfropfen $r$ stand.

Es ist unmöglich, mit dem Quecksilber so nahe an die

1) v. Wroblewski und Olszewski, Wied. Ann. 20. p. 246. 1883.

2) In der Figur nicht angegeben. 
Oberfäche des Hlüssigen Sauerstoffes zu kommen, dass die Menge des nicht verflüssigten Gases ausser Acht gelassen werden kann. Und da andererseits diese Gasmenge sich ebenso wenig wie bei den Versuchen von Pictet berechnen lässt, so kann die Dichtigkeit des Sauerstoffes aus der zum Versuche genommenen Gasmenge und aus dem Volumen der erhaltenen Flüssigkeit nicht exact berechnet werden. Die Vernachlässigung des nicht verflüssigten Gases würde die Dichtigkeit grösser erscheinen lassen, als sie in Wirklichkeit ist. Man kann sich deshalb dieser Berechnung bedienen, nur um zu sehen, was für Werthe durch die Dichtigkeit des flüssigen Sauerstoffes nicht erreicht werden können. Die auf diese Weise ausgeführten Berechnungen der Versuche, ron denen gleich unten die Rede sein wird, zeigten, dass diese Dichtigkeit, gemessen unter dem Verflüssigungsdrucke bei der Temperatur von etwa $-130^{\circ} \mathrm{C}$, kleiner als Eins sein muss, und dass sie sogar nicht den Werth ron 0,94 erreicht.

Zur Ermittelung des exacten Werthes habe ich folgende Methode erdacht.

Es sei $Q_{1}$ die zum Versuche genommene Sauerstoffmenge. Wird sie bei $-130^{\circ} \mathrm{C}$. verflüssigt und ist das Quecksilber in der Capillare bis zur Stelle $A$ gekommen, so hat man:

$$
Q_{1}=v_{1} d_{1}+q_{1} \text {, }
$$

wo $\nu_{1}$ und $d_{1}$ Volumen und Dichtigkeit des flüssigen Sauerstoffes und $q_{1}$ die nicht verflüssigte Sauerstoffmenge bedeuten, Die Dimensionen der Röhre sind so gewählt worden, dass $q_{1}$ im Vergleiche zu $v_{1} d_{1}$ sehr klein ist.

Füllt man jetzt das Rohr mit Kohlensäure oder mit Stickstoffoxydul und verflussigt man dieses Gas bei $0^{\circ}$ (wobei man mit dem Quecksilber wieder bis zur Stelle $A$ kommt), so hat man für das genommene Gas:

$$
Q_{2}=y_{2} d_{2}+q_{3} \text {, }
$$

wo ganz analog $Q_{2}$ die zum Versuche genommene Gasmenge, $\nu_{2}$ und $d_{2}$ Volumen und Dichtigkeit der erhaltenen Flissigkeit und $q_{2}$ die nicht verflüssigte Gasmenge bedeuten.

Wird (1) durch (2) dividirt und alles nach $d_{1}$ aufgelöst, so erhält man für die Dichtigkeit des Sauerstoffes: 


$$
d_{1}=d_{2} \frac{\nu_{2} Q_{1}}{\nu_{1} Q_{2}}+\frac{q_{2} Q_{1}-q_{1} Q_{2}}{v_{1} Q_{2}}
$$

Da die Verflüssigungsdrucke des Sauerstoffes bei -130 und die der Kohlensäure oder des Stickstoffoxyduls bei $0^{0}$ nicht viel voneinander verschieden sind, so ist es nicht schwer, $z u$ erkennen, dass das Glied $\left(q_{2} Q_{1}-q_{1} Q_{2}\right) / v_{1} Q_{2}$ nur sehr wenig von der Null verschieden ist und vernachlässigt werden kann.

Die Methode gestattet deshalb, mit Hülfe der Gleichung:

$$
d_{1}=d_{2} \frac{\nu_{2} Q_{1}}{\nu_{1} Q_{2}}
$$

die Dichtigkeit des Sauerstoffes mit grosser Annäherung zu bestimmen, wenn die Gasmengen und die Dimensionen der Capillare passend gewählt worden sind. Es ist nur exacte Kenntniss der Dichtigkeit des zu Hülfe genommenen Gases erforderlich.

Für Kohlensäure und Stickstoffoxydul können die im Laboratorium ron Bunsen mit grosser Sorgfalt durch Andréeff ${ }^{1}$ ) ermittelten Zạhlen benutzt werden. Andréeff fand die Dichtigkeit der Kohlensäure bei $0^{\circ}$ und unter dem Verflüssigungsdrucke gleich 0,9471 und diejenige des Stickstoffoxyduls gleich 0,9370, wobei die Dichtigkeit des Wassers bei $4^{\circ} \mathrm{C}$. gleich Eins gesetzt wurde.

Zur Prüfung der Methode habe ich die Dichtigkeit des bereits im flüssigen Zustande aus England bezogenen Stickstoffoxyduls durch Vergleich mit der Dichtigkeit der flüssigen Kohlensäure bestimmt. Ich fand sie gleich 0,9434 , woraus zu ersehen ist, in wie weit die Methode zuverlässige Werthe liefert.

$\S 3$. Zu den Beobachtungen wurden zwei Röhren benutzt. Das ganze Volumen $V$ der ersten Röhre, gemessen bei $22,73^{\circ} \mathrm{C}$. betrug $202,55 \mathrm{ccm}$, das $\nabla$ olumen $R$ eines Centimeters der Capillare 0,051793 ccm. Bei der zweiten Röhre war $V$ bei $23,44^{\circ} \mathrm{C}$. gleich $198,27 \mathrm{ccm}$ und $R 0,05282 \mathrm{ccm}$.

Die Berechnung der Versuche geschah mit Hülfe der folgenden zwei Gleichungen:

1) André eff, Lieb. Ann. 110. p. 1-16. 1859, auch Ann. de chim. et de phys. (4) 56. p. 31i-333. 1859. 


$$
d=\frac{\nabla\left(1+\beta \Theta^{\prime}\right) S \cdot 0,001293 \cdot P}{v R\left(1+\beta \Theta^{\prime \prime}\right)\left(1+\alpha \Theta^{\prime}\right) 76},
$$

wo $V$ und $R$ die bereits angegebene Bedeutung haben und

$v$ die abgelesene Länge der Flüssigkeitssäule in $\mathrm{cm}$

$\beta$ den cubischen Ausdehnungscoëfficienten des Glases $=0,0000255$,

$\alpha$ den Ausdehnungscoëfficienten des Gases $=0,00366$

(aus Bunsen's Tabellen in "Gasometrische Methoden"),

$\Theta$ und $P$ Temperatur und Druck - der letztere in Centimetern Quecksilber -, bei welchen die Röhre mit dem Gase gefüllt worden ist,

$\Theta^{\prime \prime}$ die Temperatur, bei welcher die Verflüssigung des Gases stattfindet (für den Sauerstoff $-130^{\circ} \mathrm{C}$.),

$S$ das specifische auf Luft als Einheit bezogene Gewicht des Gases,

$d$ die gesuchte Dichtigkeit

bedeuten. Diese Formel erhält man, indem man die in der Gl. (1) oder (2) angegebenen Grössen auf bekannte Weise ausdrückt, $q$ gleich 0 setzt und die Gleichung nach $d$ auflöst. Sie gestattet also, den Werth zu berechnen, welcher durch die Dichtigkeit des Sauerstoffes nicht erreicht wird.

Die zweite Formel ist:

$$
d_{1}=d_{2} \frac{\nu_{2}\left(1+\beta \Theta_{1}^{\prime}\right)\left(1+\alpha \Theta_{2}^{\prime}\right) S_{1} P_{1}}{\nu_{1}\left(1+\beta \Theta_{9}^{\prime}\right)\left(1+\beta \Theta^{\prime \prime}\right)\left(1+\alpha \Theta_{1}^{\prime}\right) S_{2} P_{9}}
$$

wo die mit dem Index Eins versehenen Buchstaben sich auf den Sauerstoff, diejenigen mit dem Index Zwei auf die Kohlensäure beziehen und dieselbe Bedeutung wie in der Gl. (4) haben. Diese Formel ist nichts weiter als die Gl. $\left(3^{a}\right)$ und liefert die gesuchte Dichtigkeit des Sauerstoffes.

Bei der Bestimmung der Länge der F'lüssigkeitssäule wurde jedesmal die Meniskushöhe ermittelt und $1 / 3$ dieser Höhe zu der am tiefsten Orte abgelesenen Länge zugezählt.

Bei den Versuchen mit der Kohlensäure wurde der nach abwärts gebogene Schenkel der Capillare bis zur Biegungsstelle $A$ in einem Gemisch ron Eis und Wasser gehalten, durch welches beständig ein Luftstrom ging. Es wurde genau darauf geachtet, dass keine Kohlensäure zwischen dem Quecksilber und dem horizontalen Theile der Capillare zurückblieb. 
Versuche mit der ersten Röhre.

Kohlensäure.

Erste Füllung (I) Zweite Füllung (II)

$$
\begin{array}{rlrl}
\Theta^{\prime} & =13,78^{\circ} \mathrm{C} . & 17,31^{\circ} \mathrm{C} . \\
P & =74,18 \mathrm{~cm} & 74,64 \mathrm{~cm} \\
v & =7,263 \mathrm{~cm} & 7,16 \mathrm{~cm}
\end{array}
$$

$$
\begin{gathered}
\text { S'auerstoff. } \\
\Theta^{\prime}=19,31^{0} \text { C. } P=74,34 \mathrm{~cm} .
\end{gathered}
$$

Erster Versuch.

$\nu$ erste Ablesung 5,413 cm zweite Ablesung 5,406 cm

$d$ nach der Gl. (4) aus der ersten Abl. 0,9471

" , zweiten , 0,9484

$d$ nach der Gl. (5) aus der ersten Abl. und I 0,9065

" " zweiten $"$ " I 0,9077

" , ersten " , II 0,8989

" " zweiten " ., II 0,9000

Zweiter Versuch.

$\nu$ erste Ablesung $5,493 \mathrm{~cm}$ zweite Ablesung $5,45 \mathrm{~cm}$

$d$ nach der Gl. (4) aus der ersten Abl. 0.9334

$" \#$ zweiten $, 0,9407$

$d$ nach der GI. (5) aus der ersten Abl. und I 0,8933

$$
\begin{aligned}
& " \text { " zweiten " . I } 0,9004 \\
& \text { ". ersten " .. II } 0,8858 \\
& \text { " " zweit'n . " } \text { II } 0,893
\end{aligned}
$$

Versuche mit der zweiten Röhre.

$$
\begin{array}{lc}
\text { Kohlensiure } & \text { Sauerstoff } \\
\Theta^{\prime}=22,02^{\circ} \mathrm{C} . & 22,55^{\circ} \mathrm{C} . \\
P=74,46 \mathrm{~cm} & 74,49 \mathrm{~cm} \\
\nu=6,577 \mathrm{~cm} & 5,00 \mathrm{~cm}
\end{array}
$$

$d$ nach der GL. (4) 0,9756 wach der Gl. (5) 0,9024

Die Capillare ist etwas verkürzt worden.

Kohlensiaure.

$$
\begin{aligned}
& \text { Erste Füllung (III) Zweite Füllung (IV) } \\
& \Theta^{\prime}=20,44^{\circ} \mathrm{C} . \quad 19,44^{\circ} \mathrm{C} \text {. } \\
& P=72,99 \mathrm{~cm} \quad 74,89 \mathrm{~cm} \\
& \nu \text { ergte Abl. } 6,46 \mathrm{~cm} \quad 6,68 \mathrm{~cm} \\
& \nu \text { zweite }, 6,48 \mathrm{~cm} \\
& \text { Sauerstoff. } \\
& \Theta^{\prime}=19,12^{\circ} \text { C. } \quad P=73,22 \mathrm{~cm} \quad \nu=5 \mathrm{~cm} \\
& d \text { nach der Gl. (4) } 0,970 \\
& d \text { nach der Gl. (5) aus der ersten Abl. von III } 0,8944 \\
& d \text { nach der Gl. (5) aus IV } 0,8983 \text {. ” " III } 0,897
\end{aligned}
$$


Stellt man alle Werthe zusammen, so hat man für die Dichtigkeit des flüssigen Sauerstoffes aus den Versuchen:

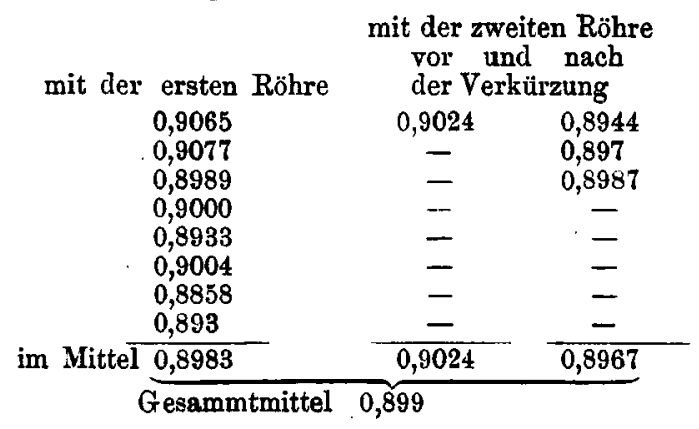

Es bleibt noch nachzusehen, in wie weit dieser Werth durch die Vernachlässigung des zweiten "Gliedes auf der rechten Seite der Gl. (3) beeinflusst wird. Zu diesem Zwecke habe ich dieses Glied für den mit der zweiten Röhre noch vor der Verkürzung der Capillare ausgeführten Versuch berechnet.

Die zum Versuche genommene Menge Kohlensäure war $179,8 \mathrm{~cm}$ bei $0^{\circ}$ und $76 \mathrm{~cm}$ Quecksilberdruck oder $0,35547 \mathrm{~g}$, diejenige des Sauerstoffes $179,54 \mathrm{ccm}$ oder $0,25667 \mathrm{~g} . \quad d_{1}$ war 0,9024 .

Das Volumen von $6,577 \mathrm{~cm}$ der Capillare reducirt auf die Temperatur des Versuches war gleich $0,3472 \mathrm{ccm}$, folglich war 0,32879 $\mathrm{g}$ das Gewicht der flüssigen, und 0,02668 $\mathrm{g}$ das der gasförmigen Kohlensäure.

Da das Volumen des flüssigen Sauerstoffes $0,26308 \mathrm{ccm}$ betrug, so enthielt die Capillare - wenn man für diese Correction den soeben angeführten Werth ron $d$ benutzt $0,2374 \mathrm{~g}$ Flüssigkeit und 0,01927 g Gas.

Daraus ergibt sich:

$\frac{q_{3} Q_{1}-q_{1} Q_{8}}{\nu_{1} Q_{3}}=\frac{0,02668 \times 0,25667-0,01927 \times 0,35547}{0,26308 \times 0,35547}=-0,000019$.

Der wahrscheinlichste Werth des specifischen Gewichtes des flussigen Sauerstoffes, gemessen bei der Temperatur von etwa - $130^{\circ}$ C. und unter dem Verflüssigungsdrucke ist mithin 0,899 . 
Zum Schluss exlaube ich mir noch zwei Bemerkungen.

Der für die Dichtigkeit des flüssigen Sauerstoffes erhaltene Werth schliesst durchaus nicht die Richtigkeit der Ansichten von Durnas aus. Der berühmte Chemiker bemerkt mit Recht in einem Zusatz ${ }^{1}$ ) zu meiner in den Comptes Rendus publicirten Note, dass die Gase im flüssigen $\mathrm{Zu}$ stande sehr grosse Ausdehnungscoëfficienten haben, und dass dem zu Folge, wenn es möglich wäre, den Sauerstoff noch weiter abzukühlen, seine Dichtigkeit immer mehr gegen Eins convergiren würde. Uebrigens - meint Hr. Dumas - entspricht der gefundene Werth einem Molecularvolumen des Sauerstoffes, welcher sich von demjenigen des Schwefels nicht weiter entfernt, als die meisten Molecularvolumina der einfachen Körper von einer und derselben Gruppe voneinander abweichen.

Die zweite Bemerkung bezieht sich auf Versuche Pictet's.

Gleich nach der Publication der ersten Note rom 16. April d. $J_{.}{ }^{2}$ ) erhielt ich mehrere Anfragen, ob nicht ein Druckfehler in den dort publicirten Verflüssigungsdrucken v.orliege; so verschieden sind diese Zahlen von denjenigen, welche Pictet aus seinen Versuchen abgeleitet hat. Den Versuchen Pictet's zu Folge wäre die Dampfspannung des flüssigen Sauerstoffes bei $-130^{\circ} \mathrm{C}$. gleich 273 Atmosphären nnd bei $-140^{\circ}$ C. gleich 252 Atmosphären. ${ }^{3}$ )

Um diese Frage zu beantworten, muss zuerst gefragt werden, ob Pictet wirklich bei seinen Versuchen die von ihm angegebenen Temperaturen gehabt hat. Eine directe Antwort darauf ist - wie dies bereits an einem anderen Orte $^{4}$ ) bemerkt worden - unmöglich. Ich habe aber versucht, mir eine Vorstellung daron auf indirectem Wege zu verschaffen. Man laann nämlich, wenn man sich der Beobachtungen am Aethylen, die ich noch nicht publicirt habe bedient, die Pictet'schen Temperaturen mit Hülfe des

1) Durnas, Compt. rend. 97. p. 168. 1883.

2) v. Wroblewski und Olzewski, Compt. rend. 96. p. 1140. 1883.

3) Pictet l. c. p. 210.

4) v. Wroblewski und Olszewski, Wied. Ann. 20. p. 255. 1883. 
Satzes von den correspondirenden Temperaturen ${ }^{1}$ ) annähernd berechnen. Andererseits kann man in die Temperaturgleichung Pictet's einige wahrscheinlichere Werthe für die darin vorkommenden Constanten einführen. Beide Wege führen zu dem Resultate, dass Pictet bei seinen Versuchen in dem abgekühlten Theil des Apparates eine Temperatur zwischen -120 und $-130^{\circ} \mathrm{C}$. gehabt hat.

Wenn man jetzt berücksichtigt, dass die kritische Tem. peratur des Sauerstoffes - so weit ich ${ }^{2}$ ) sie bis jetzt bestimmen konnte - etwa $-113^{\circ} \mathrm{C}$. und der dieser Temperatur entsprechende kritische Druck nur etwa 50 Atmosphären betragen, so unterliegt es keinem $Z_{\text {weifel, dass jedesmal zu }}$ Anfang der Pictet'schen Versuche, als der Druck-noch 470 Atmosphären betrug, der Sauerstoff in dem abgekühlten Theil des Apparates im flüssigen Zustande sich befand.

Die Kenntniss der Dichtigkeit des flüssigen Sauerstoffes gestattet jetzt, die Berechnung Offret's in umgekehrter Richtung auszuführen. Diese Berechnung ist zwar nicht streng richtig, sie gestattet aber doch eine annähernde Controle. Im Apparate von Pictet befanden sich 274,12 $\mathrm{g}$ Sauerstoff. Das Volumen der abgekühlten Röhre betrug nach der Annahme Offret's 45,5 ccm, dasjenige der erhitzten Retorte $967,5 \mathrm{ccm}$. Füllt man die Röhre mit dem Sauerstoff ron der Dichtigkeit 0,899 , so bleiben noch für den Inhalt der Retorte 233,2 g Sauerstoff, die bei der Tem. peratur ron $485^{\circ} \mathrm{C}$. - die Gültigkeit des Mariotte-Gay. Lussac'schen Gesetzes vorausgesetzt - den Druck ron 467,9 Atmosphären ausüben können. Pictet beobachtete 470 Atmosphären.

Bis so weit also wären alle aus den Versuchen von $\mathrm{Pictet}$ gezogenen Consequenzen rịchtig. Hätte Pictet zu seinen fünf Versuchen nicht immer eine und dieselbe Menge Sauerstoff genommen, die für seinen Apparat offenbar viel zu gross war, so hätte er auch niedrigere Drucke beobachten können. Leider ist bei den Pictet'schen Versuchen ron

1) Groshans. Pogg. Ann. 78. p. 112. 1849.

2) v. Wroblewski. Compt. rend. 97. p. 309-310. 1883. 
dem Augenblicke an, wo der Hahn des Apparates zum ersten mal geöffnet wird, jede weitere Berechnung unmöglich und ich muss desshalb die von ihm im Bezug auf die Dampfspannung des flüssigen Sauerstoffes gezogene Consequenz, welche im grellsten $W$ iderspruche mit den direct beobachteten Thatsachen steht, als unrichtig bezeichnen.

Physik. Inst. d. Univ. Krakau.

\section{Ueber die innere Ausdehnungsarbeit} von Flüssigkeitsgemischen im Vergleich zu derjenigen ihrer Bestandtheile; von Joseph Drecker. (IIIerzu Tat. YIII Fig. 2-7.)

Dass abweichend von dem Verhalten der Metallegirungen die specifische Würme eines Flüssigkeitsgemisches nicht allgemein gleich ist der aus den Bestandtheilen berechneten mittleren specifischen Wärme, ist durch die Arbeiten von Bussy und Buignet ${ }^{1}$ ), Dupré und Page ${ }^{2}$ ) und Schüller ${ }^{3}$ ) nachgewiesen worden. Am ausgedelintesten sind die Versuche, welche Schüller angestellt hat; er untersuchte Gemische der drei letzten Flüssigkeiten. Bei allen Gemischen, welche Alkohol enthielten, zeigte sich die beobachtete specifische Wärme grösser als die aus den Bestandtheilen berechnete, während die Differenzen zwischen beobachteter und berechneter specifischer Wärme bei den. Mischungen Chloroform-Schwefelkohlenstoff, Chloroform-Benzin und Schwefelkohlenstoff-Benzin so klein waren, dass sie vollständig innerhalb der Grenzen der unvermeidlichen Beobachtungsfehler lagen.

Hält man an dem Clausius'schen Satze von der Constanz der wahren Wärmecapacität eines Körpers fest, so muss man schliessen, dass bei Alkoholgemischen die bei der Tem-

1) Bussy et Buignet, Compt. rend. 64. p. 330. 1867.

2) Dupré et Page, Phil. Trans. 1869. p. 591.

3) Schüller, Pogg. Ann. Ergbd. 5. p. 116 u. 192.1871. 


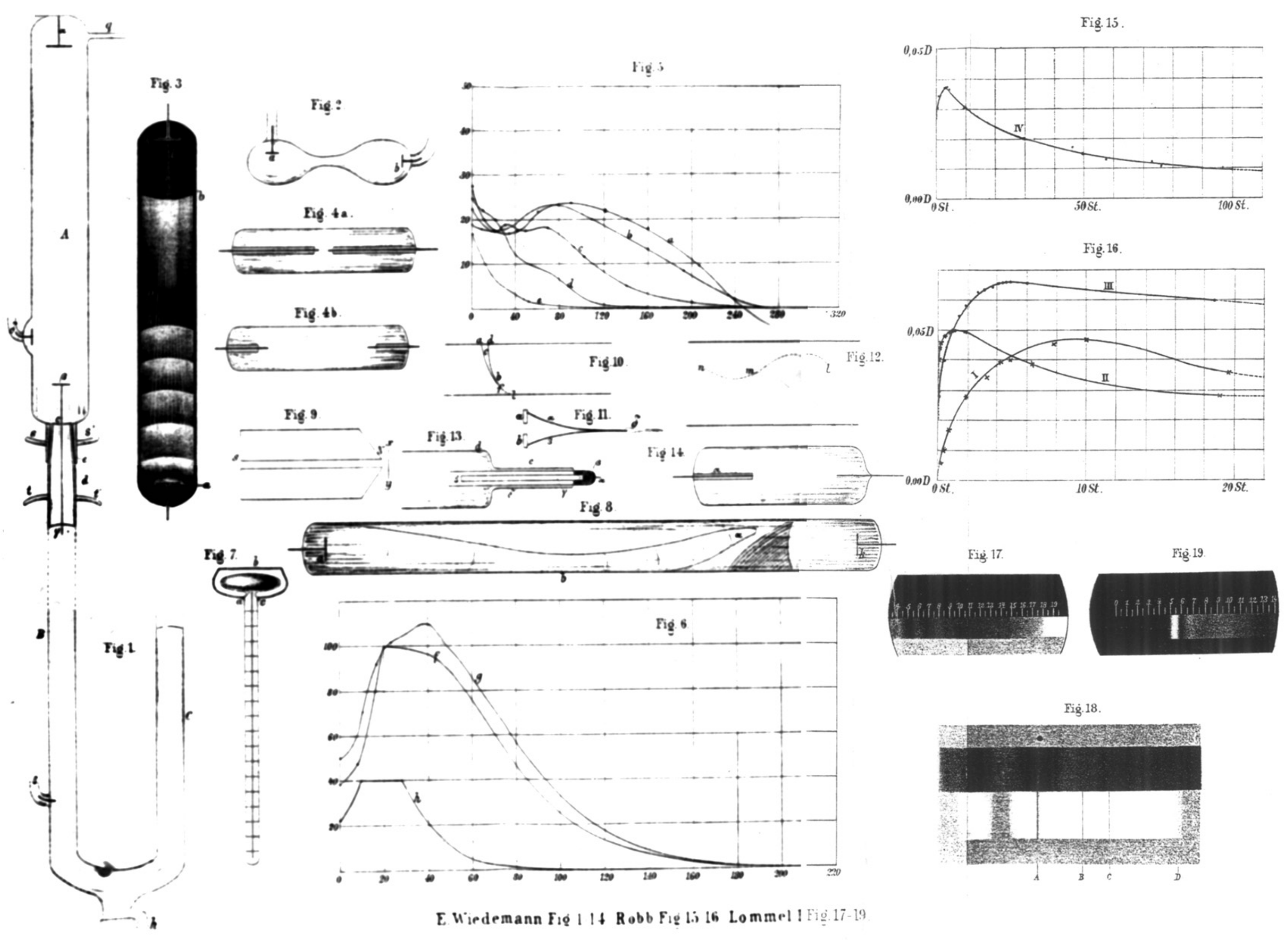

